\title{
Successful treatment of infliximab-induced psoriasiform skin lesions despite biologic therapy intensification in a paediatric patient with Crohn's disease and primary sclerosing cholangitis
}

\author{
Agata Wasilewska, Małgorzata Chmielowska-Trybek, Małgorzata Sładek \\ Department of Paediatrics, Gastroenterology and Nutrition, Faculty of Medicine, Jagiellonian University Medical College, \\ Krakow, Poland
}

\section{ABSTRACT}

Therapy with infliximab (IFX), a tumour necrosis factor- $\alpha$ (TNF- $\alpha$ ) inhibitor, has been demonstrated to be highly effective in several immune-mediated inflammatory diseases including Crohn's disease (CD) and psoriasis, both in adult and paediatric patients. With the increasingly widespread use and longer follow-up of IFX, one unexpected adverse effect that has been reported is the occurrence of psoriasis-like lesions, mainly during the maintenance therapy. The present report was aimed at describing a new case of IFX-induced psoriasis-like lesions in a 17-year-old boy with CD and primary sclerosing cholangitis (PSC), which was successfully treated with topical agents, despite IFX therapy intensification due to loss of the clinical response.

KEY WORDS:

infliximab, Crohn's disease, primary sclerosing cholangitis, paradoxical psoriasis, adverse effects.

\section{INTRODUCTION}

Selective blockade of tumour necrosis factor- $a$ (TNF- $\alpha$ ), a proinflammatory cytokine with infliximab (IFX), a chimeric anti-TNF-a monoclonal antibody, has demonstrated effectiveness in both induction and maintenance of remission in several immune-mediated inflammatory diseases including Crohn's disease (CD) and psoriasis [1]. Infliximab induces a prompt clinical response, promotes mucosal healing, lowers the risk of surgery, restores growth retardation in paediatric CD patients, and has a steroid sparing effect $[2,3]$. However, in a considerable number of patients, longterm treatment is associated with loss of the response, which requires treatment modification by increasing dose or/and frequency of drug administration to restore the clinical efficacy $[4,5]$. Treatment with IFX is well tolerated, although an increased susceptibility to infection and multiple skin lesions, such as eczema, erythema, urticaria, an exacerbation of coexisting psoriasis, paradoxical occurrence of new onset psoriasis, or psoriasiform eruptions, are increasingly reported and considered as an unwanted adverse effect of TNF- $\alpha$ blockers, mostly in the setting of rheumatologic diseases and in adults with inflammatory bowel disease [6, 7]. The pathogenesis underlying this phenomenon is not fully understood. Several theories have been proposed suggesting that an imbalance between different cytokine and activated inflammatory pathways may lead to the onset of skin lesions or exacerbation of pre-existing ones [8-12].

In adults, anti-TNF- $\alpha$-induced psoriasis is associated with stricturing and fistulising phenotypes of CD [13] tertiary IBD center were identified between 2004 and

\section{ADDRESS FOR CORRESPONDENCE:}

Małgorzata Chmielowska-Trybek, Department of Paediatrics, Gastroenterology and Nutrition, Faculty

of Medicine, Jagiellonian University Medical College, Krakow, Poland,

e-mail: malgorzata.chmielowska@gmail.com 
2016. Patients identified as having developed psoriasis while on anti-TNF underwent detailed retrospective review of dermatologic features and changes in IBD treatment prompted by the development of psoriasis. Results: Among 676 patients treated with anti-TNF (infliximab or adalimumab. Data on the association with extraintestinal manifestation is limited, although comorbid primary sclerosing cholangitis (PSC) and CD represents a unique disease phenotype with a different risk profile than that of PSC or CD alone [14]. Although cases of TNF-a-associated psoriasis have been increasingly reported in children with inflammatory bowel disease (IBD), there is a paucity of data regarding this adverse cutaneous event and PSC [14].

Herein, we report a case of a 17-year-old boy with CD and PSC, who developed IFX-induced psoriasiform skin lesions during the second year of maintenance therapy, with a good response to topical treatment, despite IFX dose intensification that was necessary for loss of the clinical response.

\section{CASE REPORT}

The case is a 17 -year-old boy, diagnosed with CD for more than 6 years, presenting with severe flare (Paediatric Crohn's Disease Index, PCDAI - 55 points) of luminal ileocolonic disease and active perianal fistula, refractory to systemic steroids and thiopurines (mercaptopurine replaced azathioprine due to leukopaenia). Additionally, PSC was diagnosed after MR cholangiopancreatography was performed. The patient was positive for atypical perinuclear antibodies (pANCA) and negative for anti-glycan antibodies including anti-Saccharomyces IgA and IgG antibodies (ASCA), anti-chitobioside IgA antibodies (ACCA), anti-laminaribioside IgG antibodies (ALCA), and anti-mannobioside IgG antibodies (AMCA). Genotyping revealed the presence of a wild type of both autophagy-related 16 like 1 (ATG16L1) and interleukin-23 receptor (IL-23R) genes and 3020insC nucleotide oligomerization domain 2 (NOD2) gene polymorphism. He had no family history of either CD or psoriasis.

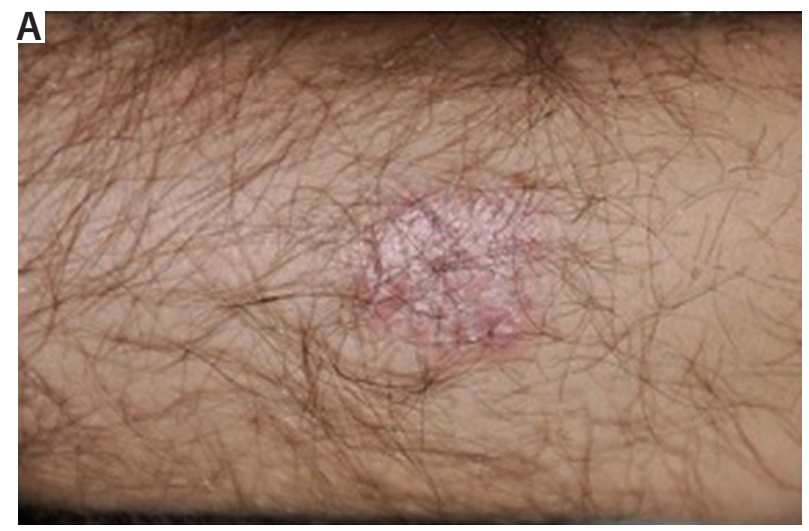

After a standard IFX induction regimen $(5 \mathrm{mg} / \mathrm{kg}$ of body weight at week 0-2-6), resulting in clinical and endoscopic remission, as well as fistula closuring, the maintenance therapy with IFX infusions every 8 week was continued. Treatment with IFX was well tolerated; however, after the $10^{\text {th }}$ infusion, the patient progressively developed psoriasis-like eruptions up to $5 \mathrm{~mm}$ in diameter that occurred in the area of the left thigh (Fig. 1B), the right knee (Fig. 1A), and in the right part of the forehead at the hair line. Skin biopsy showed lymphocyte infiltration and irregular epidermal hyperplasia of the epidermis (Fig. 2A) and parakeratosis containing neutrophils (Fig. 2B). Findings were consistent with the diagnosis of psoriasiform lesions [9]. Bacterial and fungal infections were excluded after relevant tests, as well.

After the diagnosis, a topical treatment with betamethasone and salicylic acid and cholesterol ointment was introduced, resulting in skin lesion diminishment. Therapy with IFX was continued on the standard regimen of $5 \mathrm{mg} / \mathrm{kg}$ every 8 weeks; however, after the $27^{\text {th }}$ infusion, the patient experienced moderate CD flare (PCDAI-22,5 points) with transient response to systemic steroids (methylprednisolone started at $1 \mathrm{mg} / \mathrm{kg}$ in a tapered dose). Therefore, the IFX regimen was modified by an initial dose increase to $10 \mathrm{mg} / \mathrm{kg}$, and later with subsequent shortening of the intervals between infusions to 4 weeks, resulting in restoration of the clinical response with normalisation of inflammatory biomarkers and no exacerbation of skin lesions during the next 21 months of follow-up on topical therapy.

\section{DISCUSSION}

To our knowledge, this is the first report of IFX-induced psoriasis in a paediatric patient with CD and PSC, successfully treated topically despite IFX therapy intensification.

Paradoxical psoriasis is reported in about $1.6-22 \%$ of adult IBD patients treated with TNF- $\alpha$ blockers, with a similar prevalence in children estimated at 8-13.6\% [15, 16]. Anti-TNF- $\alpha$-related skin psoriasis/psoriasiform eruptions

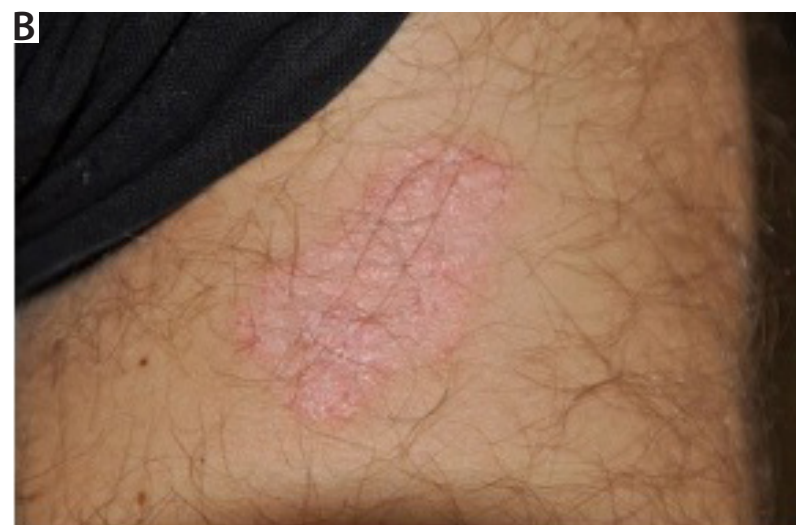

FIGURE 1. Psoriasis-like eruption: A) on the right knee; B) on the left thigh area 
A

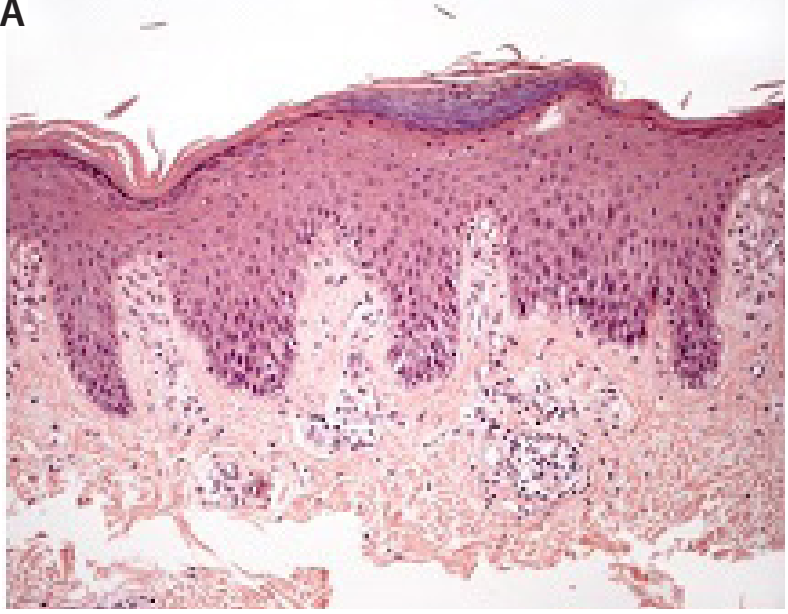

B

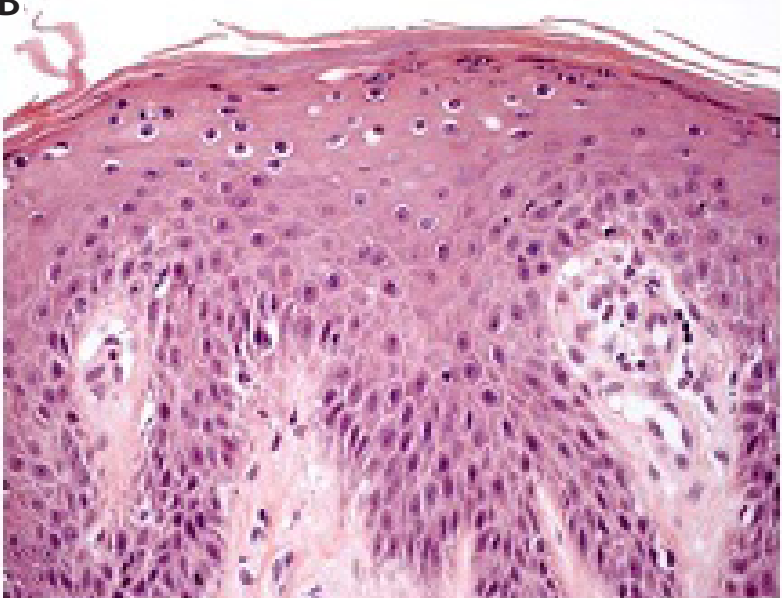

FIGURE 2. Psoriasiform reaction. A) Skin biopsy showing lymphocyte infiltration and irregular hyperplasia of the epidermis. B) Skin biopsy showing lymphocyte infiltration and paraceratosis containing neutrophils

were mostly reported in $\mathrm{CD}$ patients during the maintenance phase. The exposure time of anti-TNF- $\alpha$ agents to the onset of the skin lesion vary from the first drug administration to 48 months after initiation of treatment, with a mean onset of 20 months [17], and in most cases (93\%) they were reported to occur when CD was well controlled $[18,19]$. The latency period between initiation of IFX and the onset of skin eruptions in the presented patient was 15 months, which is consistent with the mean time period reported in the literature [16], and it occurred during maintenance therapy when the disease was well controlled. An association with perianal involvement was previously reported $[19,20]$.

Paradoxical psoriasis induced by anti-TNFs is similar in clinical presentation but differs immunologically from classical psoriasis [9]. In our patient, psoriasis-like lesions occurred in the right part of the forehead at the hair line, as well as on the lower limbs (in the area of left thigh and right knee). In the literature, clinical presentation varies from plaque psoriasis and palmoplantar pustular psoriasis occurring most commonly in adults, to psoriasiform, generalized pustular psoriasis, and inverse psoriasis [21, 22]. In children skin eruptions differ from adults, and they occur most commonly on the scalp; other areas include the face and postauricular areas, but rarely with axillar, torso, or genital involvement $[14,19]$.

The pathomechanism of this paradoxical reaction is not fully understood, but there is evidence supporting the role of increased interferon $\alpha$ (IFN- $\alpha$ ) expression as a result of TNF- $\alpha$ blockade [10]. A key role of TNF- $\alpha$ in triggering the inflammatory response is very well established; however, some TNF- $\alpha$-mediated pathways remain unknown. Published data show that a decreased level of TNF- $\alpha$, as a result of its blockade, promotes accumulation of plasmacytoid dendritic cells (pDCs), simultaneously inhibiting their maturation, which in consequence induces over-expression of skin IFN- $\alpha$ produced by pDCs. It also promotes keratinocytes to produce interferon (IFN), mostly IFN- $\beta[11,23]$.
The same up-regulation phenomena are seen in the early phases of psoriasis, but during the development of chronicity the IFN- $\alpha$ level is significantly lowered [11]. In paradoxical psoriasis, IFN-driven immune response fails to elicit T-cell autoimmunity, which differs from classical psoriasis characterised by a redundant role of T-cells [9]. The role of the interleukin (IL)-23/T-helper (TH)-17 axis has also been implicated in the pathogenesis of TNF inhibitor-induced psoriasis. Bonding of IL-17 to its receptors is a known stimulator of hyperproliferation, differentiation of keratinocytes, and recruitment of immune cells in psoriatic lesions. Interleukin 17 is produced by $\mathrm{TH}-17$, differentiation and activation of which is due to the presence of IL-23. This mechanism may be supported by a good response of psoriatic lesions to IL-12/IL-23 blocker [12, 21, 24].

There are many genes predisposing the patient to develop immunologically-mediated inflammatory diseases like $\mathrm{CD}$ or psoriasis. The most common and important genetic risk factors for $\mathrm{CD}$ are mutations in the NOD2 gene that encodes the NOD2 oligomerization domain, a cytosolic receptor that mediates innate immune response triggering proinflammatory cascade. No data were found to confirm NOD2/caspase recruitment domain family, member 15 (CARD15) susceptibility for psoriasis and psoriatic arthritis. The patient was positive for the polymorphism in the NOD2 gene, making him more susceptible to $\mathrm{CD}$, and presented with a wild type of ATGL16 and IL23R (Arg381Gly). Reports in the literature on the potential association between IFX-induced psoriasis skin lesions and IL-23 receptor polymorphism are inconclusive. Sherlock et al. reported an association in 18 paediatric patients with specific polymorphism in the IL23R gene (rs10489628, rs10789229, and rs1343151), while a Belgium group studying the IL23R Arg381Gly variant was unable to demonstrate an association with anti-TNF- $\alpha$-induced psoriasis/psoriasiform lesions [25]. IL-23 is a known proinflammatory cytokine promoting activation of lymphocyte $\mathrm{TH}-17$, which plays a role in 
the pathogenesis of chronic inflammatory disorders like $\mathrm{CD}$ or psoriasis $[11,21]$.

A wide range of therapeutic approaches have been used to manage anti-TNF- $\alpha$-induced psoriasis/psoriasiform skin lesions, but no guideline has been published so far for IBD patients. Therefore, the decision has to be made individually, depending on the severity of skin lesions and activity of the underling IBD. As described, skin eruptions in the presented patient were effectively controlled with topical treatment, making it possible to continue biological therapy despite the necessity of intensification due to loss of response. Topical therapy with continuation of treatment with TNF- $\alpha$ blockers was effective for $71 \%$ of paediatric patients reported by Eickstaedt et al. [17] and for all cases presented in the study by Courbette et al. [19]. Discontinuation of anti-TNF- $\alpha$ therapy may aggravate bowel manifestation; therefore, in patients with mild skin lesions responsive to topical agents and phototherapy, continuation of biological treatment may be possible. The use of immune suppressants, mainly methotrexate, could be another other option for patients who fail to improve on topical treatment $[6,21]$. In more severe cases, cessation of anti-TNF- $\alpha$ inhibitors might be necessary because switching to another TNF blocker is imposed with high risk of recurrence (up to $52 \%)$ [18]. Because a role of the IL-23/Th-17 axis in paradoxical psoriasis has been identified, another treatment option appeared on the horizon: ustekinumab, an antiIL-12/IL-23 antibody, has been reported to be effective in the resolution of skin lesions in more than $75 \%$ of IBD patients $[18,21]$.

We present this case to highlight that in IFX-induced paradoxical psoriasis, an individual treatment option should be considered, depending on both the severity of the underlying $\mathrm{CD}$, the intensity of skin lesions, and the response to treatment. Even the intensification of IFX therapy to control $\mathrm{CD}$ was possible because the patient responded to dermatological treatment.

\section{DISCLOSURE}

The authors declare no conflict of interest.

\section{REFERENCES}

1. Osamura A, Suzuki Y. Fourteen-year anti-TNF therapy in Crohn's disease patients: clinical characteristics and predictive factors. Dig Dis Sci 2018; 63: 204-208.

2. Church PC, Guan J, Walters TD, et al. Infliximab maintains durable response and facilitates catch-up growth in luminal pediatric Crohn's disease. Inflamm Bowel Dis 2014; 20: 1177-1186.

3. Hyams J, Crandall W, Kugathasan S, et al. Induction and maintenance infliximab therapy for the treatment of moderate-to-severe crohn's disease in children. Gastroenterology 2007; 132: 863-873.

4. De Bie CI, Hummel TZ, Kindermann A, et al. The duration of effect of infliximab maintenance treatment in paediatric Crohn's disease is limited. Aliment Pharmacol Ther 2011; 33: 243-250.
5. Van Rheenen H, Van Rheenen PF. Long-term efficacy of anti-tumor necrosis factor agents in pediatric luminal Crohn's disease: A systematic review of real-world evidence studies. Pediatr Gastroenterol Hepatol Nutr 2020; 23: 121-131.

6. Eligius Hellström A, Färkkilä M, Kolho K-L. Infliximab-induced skin manifestations in patients with inflammatory bowel disease. Scand J Gastroenterol 2016; 51: 563-571.

7. Bae JM, Lee HH, Lee BI, et al. Incidence of psoriasiform diseases secondary to tumour necrosis factor antagonists in patients with inflammatory bowel disease: a nationwide population-based cohort study. Aliment Pharmacol Ther 2018; 48: 196-205.

8. Bassukas I, Gaitanis G, Katsanos K, et al. Psoriasis and inflammatory bowel disease: links and risks. Psoriasis Targets Ther 2016; 6: 73-92.

9. Mylonas A, Conrad C. Psoriasis: Classical vs. Paradoxical. The YinYang of TNF and Type I Interferon. Front Immunol 2018; 9: 2746.

10. Conrad C, Di Domizio J, Mylonas A, et al. TNF blockade induces a dysregulated type I interferon response without autoimmunity in paradoxical psoriasis. Nat Commun 2018; 9: 1-11.

11. Fania L, Morelli M, Scarponi C, et al. Paradoxical psoriasis induced by TNF- $a$ blockade shows immunological features typical of the early phase of psoriasis development. J Pathol Clin Res 2020; 6: 55-68.

12. Stoffel E, Maier H, Riedl E, et al. Analysis of anti-tumour necrosis factor-induced skin lesions reveals strong $\mathrm{T}$ helper 1 activation with some distinct immunological characteristics. Br J Dermatol 2018; 178: 1151-1162.

13. Weizman AV, Sharma R, Afzal NM, et al. Stricturing and fistulizing Crohn's disease is associated with anti-tumor necrosis factor-induced psoriasis in patients with inflammatory bowel disease. Dig Dis Sci 2018; 63: 2430-2438.

14. Palmela C, Peerani F, Castaneda D, et al. Inflammatory bowel disease and primary sclerosing cholangitis: a review of the phenotype and associated specific features. Gut Liver 2017; 12: 17-29.

15. Mälkönen T, Wikström A, Heiskanen $\mathrm{K}$, et al. Skin reactions during Anti-TNFa therapy for pediatric inflammatory bowel disease: a 2-year prospective study. Inflamm Bowel Dis 2014; 20: 1309-1315.

16. Romiti R, Araujo KM, Steinwurz F, et al. Anti-tumor necrosis factor a-related psoriatic lesions in children with inflammatory bowel disease: Case report and systematic literature review. Pediatr Dermatol 2016; 33: 174-178.

17. Eickstaedt JB, Killpack L, Tung J, et al. Psoriasis and psoriasiform eruptions in pediatric patients with inflammatory bowel disease treated with anti-tumor necrosis factor alpha. Agents Pediatr Dermatol 2017; 34: 253-260.

18. Shivaji UN, Sharratt CL, Thomas T, et al. Review article: managing the adverse events caused by anti-TNF therapy in inflammatory bowel disease. Aliment Pharmacol Ther 2019; 49: 664-680.

19. Courbette O, Aupiais C, Viala J, et al. Infliximab paradoxical psoriasis in a cohort of children with inflammatory bowel disease. J Pediatr Gastroenterol Nutr 2019; 69: 189-193.

20. Barthel C, Biedermann L, Frei P, et al. Induction or exacerbation of psoriasis in patients with crohn's disease under treatment with anti-TNF antibodies. Digestion 2014; 89: 209-215.

21. Li SJ, Perez-Chada LM, Merola JF. TNF Inhibitor-Induced Psoriasis: Proposed Algorithm for Treatment and Management. Journal of Psoriasis and Psoriatic Arthritis 2019; 4: 70-80.

22. Iborra M, Beltrán B, Bastida G, et al. Infliximab and adalimumab-induced psoriasis in Crohn's disease: A paradoxical side effect. J Crohn's Colitis 2011; 5: 157-161.

23. Mocci G, Marzo M, Papa A, et al. Dermatological adverse reactions during anti-TNF treatments: Focus on inflammatory bowel disease. J Crohn's Colitis 2013; 7: 769-779. 
24. Fu Y, Lee CH, Chi CC. Association of psoriasis with inflammatory bowel disease: a systematic review and meta-analysis. JAMA Dermatology 2018; 154: 1417-1427.

25. Sherlock ME, Walters T, Tabbers MM, et al. Infliximab-induced psoriasis and psoriasiform skin lesions in pediatric Crohn disease and a potential association with IL-23 receptor polymorphisms. J Pediatr Gastroenterol Nutr 2013; 56: 512-518.

26. Seneschal J, Milpied B, Vergier B, et al. Cytokine imbalance with increased production of interferon- $\alpha$ in psoriasiform eruptions associated with antitumour necrosis factor- $\alpha$ treatments. Br J Dermatol 2009; 161: 1081-1088. 